im Institut für Medien- und Kommunikationswissenschaft e. V. in Münster/Werther, dessen Vorsitz er innehat.

Aber was sind schon 60 Jahre. Hat die Arbeit nicht gerade erst angefangen? Ist der Berg der wissenschaftlichen Probleme etwa schon abgetragen? Gibt es nicht tausend neue aufregende Fragen, die herausfordern und antreiben? Hans Dieter Kübler dürfte solche Fragen alle mit Ja beantworten.

WERNER FAULSTICH

\title{
Beate Schneider 60 Jahre
}

Am 15. Mai 2007 hat Beate Schneider, Professorin für Medienwissenschaft, Schwerpunkt organisatorische, rechtliche und wirtschaftliche Grundlagen von Massenmedien, am Institut für Journalistik und Kommunikationsforschung der Hochschule für Musik und Theater in Hannover, ihren 60. Geburtstag gefeiert.

Beate Schneider studierte Politikwissenschaft, Publizistik und Geschichte an der Johannes Gutenberg-Universität in Mainz und der Johann Wolfgang Goethe-Universität in Frankfurt am Main; ein Aufenthalt an der Graduate School der University of Arizona in Tucson rundete die studentische Zeit ab. 1973 promovierte sie zu einer Fragestellung, die sie viele Jahre später auf anderer Ebene noch einmal stark beschäftigte: Sie setzte sich mit dem Thema »Die politische Krise als Faktor von Integration und Entfremdung. Eine transaktionale Analyse innerdeutscher Beziehungen« auseinander. Daran anschließend arbeitete sie zwölf Jahre an der Universität der Bundeswehr in Hamburg, bevor sie ihre wissenschaftliche Heimat nach Hannover verlegte.

Seit 1985 ist Beate Schneider am hannoverschen Institut für Journalistik und Kommunikationsforschung. Sie hat dieses Institut gemeinsam mit dem Kollegen Klaus Schönbach gegründet und seitdem entscheidend geprägt. Mit viel Begeisterung und Sinn für die gesellschaftliche Bedeutung einer fundierten akademischen Ausbildung von Journalisten begann sie ihre Tätigkeit als Professorin für Journalistik, Schwerpunkt vergleichende Medienlehre. Der damals gegründete Ergänzungsstudiengang für Journalistik erschien als geeigneter Weg, die Bedürfnisse des Marktes zu befriedigen und gleichzeitig qualitative Maßstäbe zu setzen.

Auch der 1990 gegründete Studiengang Medienmanagement, damals der erste mit dieser Nomination, zeigte die akademische Grundeinstellung Beate Schneiders, nämlich den Wunsch, eine wissenschaftlich fundierte und hochwertige Ausbildung mit den Grundfragen und Anliegen der Praxis zu verbinden. Diesem Grundgedanken lassen sich die zahlreichen hochschulinternen und hochschulpolitischen Aktivitäten zuordnen, in der Zeit als Vizepräsidentin der Hochschule ebenso wie als Direktorin des Instituts. Wissenschaftlicher Pragmatismus und gleichzeitig die Bereitschaft, für unverzichtbare Anforderungen zu kämpfen, zeichnen sie innerhalb der Hochschule aus.

Diese Eigenschaften sind auch für ihre akademischen Publikationen kennzeichnend. Den Arbeiten von Beate Schneider liegt im Kern jeweils die Frage zugrunde, was die Kommunikationswissenschaft für die Medienpraxis leisten kann - und wo die Medienpraxis gut beraten wäre, auf die Wissenschaft zuzugehen und ihr zuzuhören. Inhaltlich lassen sich drei Schwerpunkte ihrer Arbeit herausstellen: Es sind dies Fragen zum Verhältnis von Medien und Politik, Fragen von Medien im Wandel und der Zusammenhang zwischen Journalismus und Gesellschaft. Exemplarisch zeigt sich dies in ihren Arbeiten zum (ost-)deutschen Mediensystem nach der Wiedervereinigung, den europäischen Pressemärkten und ihren Arbeiten zum journalistischen Aufgaben- und Selbstverständnis. Und darüber hinaus zeigen die Publikationen und die Vielzahl an Gutachten und Projektarbeiten bis heute ihre wissenschaftliche Neugier und die Bereitschaft, sich aktueller und praxisbezogener Problemen empirisch anzunehmen, um daraus Handlungsempfehlungen und Konsequenzen ableiten zu können. Dieser Wunsch nach Aufspüren, Verändern und Wirken ist ihr innerer Antrieb, der vielen Kollegen und Studierenden als Vorbild galt und gilt.

WIEBKE MÖHRING 